\title{
Prognostic factors associated with survival in patients with hepatocellular carcinoma undergoing transarterial chemoembolisation: an Australian multicenter cohort study
}

\author{
Gauri Mishra ${ }^{1,2}$, Anouk Devi,2, Eldho Paul ${ }^{3}$, William Kemp ${ }^{4}$, Ammar Majeed $^{4}$, John Lubel ${ }^{4}$, Sally Bell', Paul \\ Gow $^{5}$, Amanda Nicoll ${ }^{6}$, Siddharth Sood ${ }^{7}$, Alex Thompson ${ }^{8}$, Marno Ryan ${ }^{8}$, Stuart K. Roberts ${ }^{2,3}$ \\ 'Department of Gastroenterology, Monash Medical Centre, Melbourne 3168, Australia. \\ ${ }^{2}$ School of Medicine, Monash University, Melbourne 3800, Australia. \\ ${ }^{3}$ School of Public Health and Preventive Medicine, Monash University, Melbourne 3800, Australia. \\ ${ }^{4}$ Department of Gastroenterology and Hepatology, Alfred Health, Melbourne 3004, Australia. \\ ${ }^{5}$ Department of Gastroenterology, Austin Health, Melbourne 3084, Australia. \\ ${ }^{6}$ Department of Gastroenterology, Eastern Health, Melbourne 3128, Australia. \\ ${ }^{7}$ Department of Gastroenterology, Royal Melbourne Hospital, Melbourne 3050, Australia. \\ ${ }^{8}$ Department of Gastroenterology, St. Vincent's Hospital, Melbourne 3065, Australia.

\begin{abstract}
Correspondence to: Dr. Gauri Mishra, Department of Gastroenterology, Monash Medical Centre, 246 Clayton Rd, Melbourne
\end{abstract} \\ 3168, Australia. E-mail: Gauri.Mishra@monashhealth.org \\ How to cite this article: Mishra G, Dev A, Paul E, Kemp W, Majeed A, Lubel J, Bell S, Gow P, Nicoll A, Sood S, Thompson A, Ryan \\ $M$, Roberts SK. Prognostic factors associated with survival in patients with hepatocellular carcinoma undergoing transarterial \\ chemoembolisation: an Australian multicenter cohort study. Hepatoma Res 2021;7:56. https://dx.doi.org/10.20517/2394- \\ 5079.2021 .37
}

Received: 17 Mar 2021 First Decision: 30 Jun 2021 Revised: 6 Jul 2021 Accepted: 19 Jul 2021 First online: 29 Jul 2021

Academic Editor: Guang-Wen Cao Copy Editor: Yue-Yue Zhang Production Editor: Yue-Yue Zhang

\begin{abstract}
Aim: Transarterial chemoembolisation (TACE) is recommended therapy for intermediate-stage hepatocellular carcinoma (HCC). However, the wide variations in outcomes reflect significant heterogeneity of this patient group. We evaluated the prognostic factors associated with survival in a real-world setting to identify those at high risk of a poor outcome.

Methods: Patients with HCC who underwent initial TACE at six tertiary hospitals between 2009 to 2014 were included via an extensive search of hospital databases and electronic medical records. Overall survival (OS) was measured from the date of initial treatment to the date of death or last follow-up. Univariate and multivariate Cox regression analyses were used to assess the effects of baseline variables on post-TACE survival.
\end{abstract}


Results: The majority of the 431 eligible patients were Caucasian (80\%), male (87\%), with a mean age of 66 years and had alcohol-related cirrhosis (43\%). Most were Child-Pugh A (69\%) with BCLC stage A (59\%) or B (35\%) disease, with a median OS of 28 months. On multivariate analysis, pre-treatment ascites $(P=0.001)$ and larger HCC $(P<0.001)$ were associated with worse overall survival, while higher serum albumin $(P<0.001)$ and HBV $(P$ $=0.005$ ) were associated with improved survival.

Conclusion: Patients with advanced liver disease, including the presence of ascites and lower serum albumin, as well as those with greater tumour burden, have poorer outcomes following TACE treatment. Such findings provide a better understanding of the variation in survival after TACE and are helpful in facilitating selection and timely stage migration of patients undergoing this therapy.

Keywords: Liver Cancer, tumour stage, unresectable hepatocellular carcinoma, transarterial chemoembolisation, treatment allocation, patient selection, treatment outcomes, prognostic factors, aetiology of liver disease, chronic hepatitis B infection, cirrhosis severity

\section{INTRODUCTION}

Globally, HCC is the fourth most common cancer and the second-highest cause of cancer death worldwide $^{[1,2]}$. The severity of chronic liver disease is a key factor influencing overall survival in HCC, as unlike other solid tumours, significant liver dysfunction can preclude patients from treatment because of increased risks of adverse outcomes, including liver failure ${ }^{[3,4]}$. In addition, the stage of liver disease at presentation influences prognosis and treatment options. In this context, the Barcelona Clinic Liver Cancer (BCLC) staging system is one of the most widely utilised treatment algorithms as it incorporates both tumour staging and liver function parameters that influence patient survival ${ }^{[5-7]}$.

The majority of patients with HCC present with intermediate (BCLC B) stage disease at diagnosis ${ }^{[5,8,9]}$. According to the BCLC system, transarterial chemoembolisation (TACE) is the recommended treatment for this group based on a meta-analysis of randomised controlled trials ${ }^{[9,10]}$. In addition, as we have previously shown, up to $40 \%$ of patients with early-stage disease may receive TACE as first-line therapy because of unsuitability for resection or ablation ${ }^{[11]}$. Notably, patients with intermediate-stage disease may have asymptomatic large or multifocal intrahepatic disease in the absence of macrovascular invasion and extrahepatic metastases. In this context, the outcomes of patients having TACE are quite heterogeneous, with median survival rates varying from 20-25 months in randomised controlled trials ${ }^{[12,13]}$ to $40-45$ months in more recent prospective cohort studies from Asia ${ }^{[14]}$ and Europe ${ }^{[15]}$. Such heterogeneity in survival outcomes reported across studies may be due to the wide spectrum of tumor burden and liver dysfunction observed in patients within the BCLC stage B classification. Thus, to better understand which patients are likely to benefit from TACE, we performed a multicenter study in a real-world setting to identify pretreatment factors associated with improved survival after TACE.

\section{METHODS}

\section{Study population}

In this multicentre, retrospective real-world study, we identified patients undergoing TACE treatment for HCC from six tertiary centers in Melbourne, Australia, between January 2009 and December 2014. Patients were included if they were classified as BCLC A, B, or C with relatively well preserved European Cooperative Oncology Group (ECOG) performance status of 0-2. Patients were excluded if they received TACE as a bridge to liver transplantation or if they had TACE for any diagnosis other than HCC. All included cases had undergone review at tertiary hospital multidisciplinary meetings and were recommended to receive TACE. The diagnosis of HCC was based on imaging criteria or histology 
according to the current AASLD HCC management guidelines ${ }^{[16]}$, and the presence of cirrhosis based on biochemical and radiological criteria as described previously ${ }^{[1,1,1]}$. Each participating institution had prospectively recorded clinical decisions and treatment outcomes on hospital-specific databases for all patients with HCC who had undergone TACE. This study was approved by the Institutional Ethics Committees of each participating center. All patient data were de-identified prior to collation and statistical analysis.

\section{TACE treatment}

TACE was delivered on-demand at each tertiary center according to the local Interventional Radiologist expertise using a combination of two to three chemotherapeutic agents as either an emulsion with or without lipiodol (conventional TACE) or via drug-eluting beads with doxorubicin (DEB-TACE) as previously described ${ }^{[11]}$. Transarterial embolization (TAE) without chemotherapy was also carried out in some centers. The method of TACE delivery, degree of cannulation of hepatic artery branches, and utilisation of embolisation agents such as gelfoam or polyvinyl acetate particles was also recorded. Post TACE assessment of therapy response by imaging, based on the mRECIST criteria of the target lesion, was performed by two radiologists at each center. Laboratory and clinical parameters following TACE were also recorded, along with details of complications within four weeks of treatment, including death, post-TACE syndrome, and decompensation ${ }^{[18]}$.

\section{Data items and extraction}

Data were collected and extracted via direct chart review of each institutional prospectively recorded HCC database and electronic and paper records of eligible cases using pre-defined data points and definitions. Demographic data collected included age, gender, and country of birth, in addition to detailed information regarding the etiology and severity of underlying liver disease. Tumor-specific and related variables recorded included performance status, the number of tumors $(1 / 2 / 3 />3)$, size of the largest lesions, macrovascular invasion, extrahepatic spread derived directly from pre-treatment and diagnostic imaging, serum alfa-fetoprotein levels, and BCLC staging.

\section{Primary endpoint}

Overall survival was defined from the date of first TACE/TAE to death or last clinical follow-up to the 31 st January 2019. These data were derived from hospital-based electronic records and further cross-referenced with records from the Victorian Births, Deaths, and Marriages registry as previously described ${ }^{[17]}$.

\section{Statistical analysis}

Categorical variables were summarised using counts and percentages. According to data type and distribution, continuous variables were summarised using means and standard deviations or medians and interquartile ranges (IQR). The Kaplan-Meier product-limit method was used to plot survival as a function of time after treatment, and comparisons between curves were made with the log-rank test. Univariate and multivariate analyses were performed using Cox proportional hazards regression to assess the effects of clinical, liver disease, and tumor variables before the initial TACE therapy on overall survival, with results reported as hazard ratios and $95 \%$ confidence intervals. Variables with $P \leq 0.05$ in the univariate analysis or those judged to be clinically significant were considered as candidate variables for inclusion in a hierarchical regression model to identify the independent predictors of overall survival.

A sub-group analysis was performed on a refined cohort of patients derived from the overall cohort with BCLC stage A or B disease who had TACE monotherapy without combination use of other treatment modalities such as radiofrequency ablation or surgical resection [Figure 1]. This subgroup excluded ChildPugh C liver disease, main branch portal vein invasion, and poor performance status prior to TACE (ECOG 


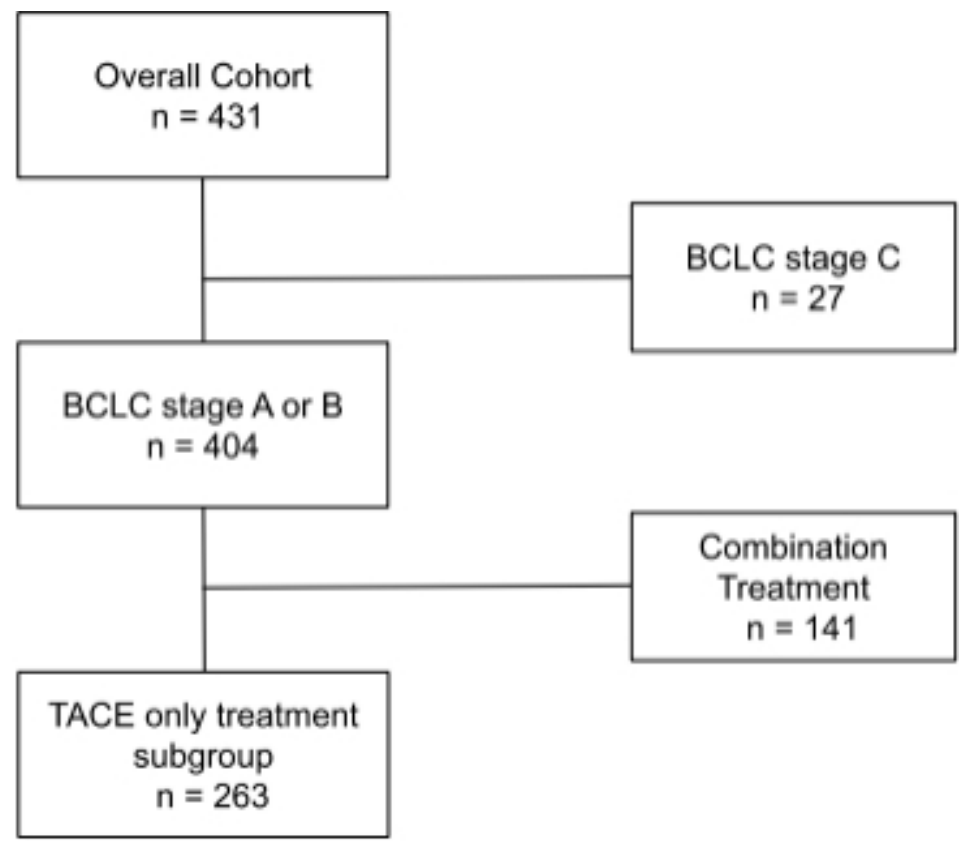

Figure 1. Flow chart for patients included in TACE only treatment subgroup. BCLC: Barcelona clinic liver cancer; TACE: transarterial chemoembolization.

$\geq 2$ ) in order to assess the impact of treatment within current guidelines compared to current practice encapsulated within the overall cohort. All reported $P$-values are two-sided, and a $P<0.05$ was chosen to indicate statistical significance.

\section{RESULTS}

\section{Study population}

Between January 2009 to December 2014, 431 patients who underwent TACE for hepatocellular carcinoma across the six participating centers were eligible for inclusion in this study [Table 1]. Most patients were cirrhotic (92\%) and had portal hypertension (86\%) at the time of diagnosis. These patients mainly had (61\%) compensated Child-Pugh A cirrhosis, with a smaller proportion (30\%) having Child-Pugh B liver disease. The vast majority had BCLC stage A (59\%) and stage B (35\%) disease with a small proportion having BCLC stage C (6\%) disease.

Baseline characteristics, including etiology of underlying chronic liver disease and pre-treatment tumor number, size, and macrovascular invasion, were recorded [Table 1]. A total of 141 patients had other treatment modalities administered in combination with TACE, including surgical resection and local ablation [Table 1]. Conventional TACE was performed in the majority ( $81 \%$ ) of patients with a smaller subset undergoing DEB-TACE (19\%), while selective catheterisation was performed in two-thirds of cases. Most patients underwent at least two cycles of TACE, with a few $(n=12)$ individuals undergoing greater than five cycles. The refined subgroup comprised 263 patients with BCLC A (61\%) and BCLC B (39\%) stage HCC who had undergone TACE alone.

\section{Overall survival}

From January 2009 to January 2019, 333 (77\%) of patients had died, with the remaining patients' follow up censored at the date of last clinical follow-up. The median OS of the group was 28 (IQR: 14-51) months, with a wide range of survival outcomes observed of between 1 day and 110 months [Figure 2]. The median 
Table 1. Cohort Characteristics at baseline and post initial TACE therapy in the overall cohort

\begin{tabular}{|c|c|}
\hline \multicolumn{2}{|c|}{ Baseline characteristics of overall cohort $(n=431)$} \\
\hline Age (years), mean (SD) & $66(11)$ \\
\hline Male, $n(\%)$ & $376(87)$ \\
\hline Female, $n(\%)$ & $55(13)$ \\
\hline \multicolumn{2}{|l|}{ Ethnicity, n (\%) } \\
\hline Caucasian & $343(80)$ \\
\hline Asian & $68(16)$ \\
\hline Other & $20(5)$ \\
\hline \multicolumn{2}{|l|}{ Aetiology of Liver disease, $n$ (\%) } \\
\hline $\mathrm{HCV}$ & $167(39)$ \\
\hline HBV & $82(19)$ \\
\hline NAFLD & $105(24)$ \\
\hline Alcohol & $186(43)$ \\
\hline Haemochromatosis & $15(3)$ \\
\hline Other & $18(4)$ \\
\hline BMI, mean (SD) & $27(5)$ \\
\hline \multicolumn{2}{|l|}{ Serum markers, median (IQR) } \\
\hline Haemogloblin, $\mathrm{g} / \mathrm{L}$ & $136(122-147)$ \\
\hline Platelets, 10^9/L & $119(81-167)$ \\
\hline $\mathrm{AFP}, \mathrm{ng} / \mathrm{mL}$ & $17(5-125)$ \\
\hline $\mathrm{ALT}, \mathrm{U} / \mathrm{L}$ & $46(29-76)$ \\
\hline Albumin, $\mathrm{g} / \mathrm{L}$ & $35(31-39)$ \\
\hline Bilirubin, $\mu \mathrm{mol} / \mathrm{L}$ & $17(12-27)$ \\
\hline INR & $0.9(1.0-1.3)$ \\
\hline Creatinine, $\mu \mathrm{mol} / \mathrm{L}$ & $76(66-90)$ \\
\hline $\mathrm{Na}, \mathrm{mmol} / \mathrm{L}$ & $139(137-140)$ \\
\hline \multicolumn{2}{|l|}{ Liver function, $n(\%)$} \\
\hline Portal HTN/Ascites/HE & $371 / 62 / 22(86 / 14 / 5)$ \\
\hline Child Pugh grade (A/B) & $264 / 129(61 / 30)$ \\
\hline MELD score & $9(7-12)$ \\
\hline $\operatorname{ECOG}(0 / 1 / 2 / 3), n(\%)$ & $230 / 163 / 35(53 / 38 / 8)$ \\
\hline \multicolumn{2}{|l|}{ Tumour characteristics } \\
\hline Tumour nodules $(1 / 2 / 3 />3)$ & $196 / 93 / 36 / 106(45 / 22 / 8 / 25)$ \\
\hline Tumour size, cm (median, IQR) & $3.4(2.1-5.0)$ \\
\hline$M V I, n(\%)$ & $16(4)$ \\
\hline$E H S, n(\%)$ & $12(3)$ \\
\hline BCLC stage $(A / B / C), n(\%)$ & $253 / 151 / 27(59 / 35 / 6)$ \\
\hline \multicolumn{2}{|l|}{ Previous treatment, $n(\%)$} \\
\hline Resection /Ablation/PEI, n (\%) & $29 / 42 / 7(7 / 10 / 2)$ \\
\hline TACE treatments $(1 / 2 / 3 />3), n(\%)$ & 138/132/80/81 (32/31/19/9) \\
\hline Type (cTACE/DEB TACE/TAE) & $337 / 89 / 4(78 / 21 / 1)$ \\
\hline \multicolumn{2}{|l|}{ Selectivity } \\
\hline selective/superselective/non selective & 285/77/61 (66/18/14) \\
\hline \multicolumn{2}{|l|}{ Post TACE, $n(\%)$} \\
\hline \multicolumn{2}{|l|}{ mRECIST response } \\
\hline CR/PR & 137/158/ (32/37) \\
\hline $\mathrm{SD}^{\dagger} / \mathrm{PD}$ & $43 / 39(10 / 9)$ \\
\hline Adverse events & $94(22)$ \\
\hline Death & $2(0.5)$ \\
\hline PTS/decompensation & $52 / 16(12 / 4)$ \\
\hline
\end{tabular}


Renal dysfunction/other

Post TACE treatment

Resection /ablation/ PEI

Sorafenib
$9 / 24(2 / 6)$

$16 / 35 / 12(4 / 8 / 3)$

100 (23)

AFP: Alpha-fetoprotein; ALT: alanine transaminase; BCLC: Barcelona clinic liver cancer; CTACE: conventional TACE; DEBTACE: drug eluting bead TACE; ECOG: Eastern cooperative oncology group; EHS: extra hepatic spread; HCV: hepatitis C virus; HBV: hepatitis B virus; INR: international normalized ratio; mRECIST: modified response evaluation criteria in solid tumors; MVI: macrovascular invasion; N/A: not applicable; NAFLD: non alcoholic fatty liver disease; OS: overall survival; PD: progressive disease; PEl: percutaneous ethanol injection; Portal HTN: portal hypertension; PR: partial response; PTS: post TACE syndrome; SD: standard deviation; SD": stable disease; TACE: transarterial chemoembolization; TAE: transarterial embolization.

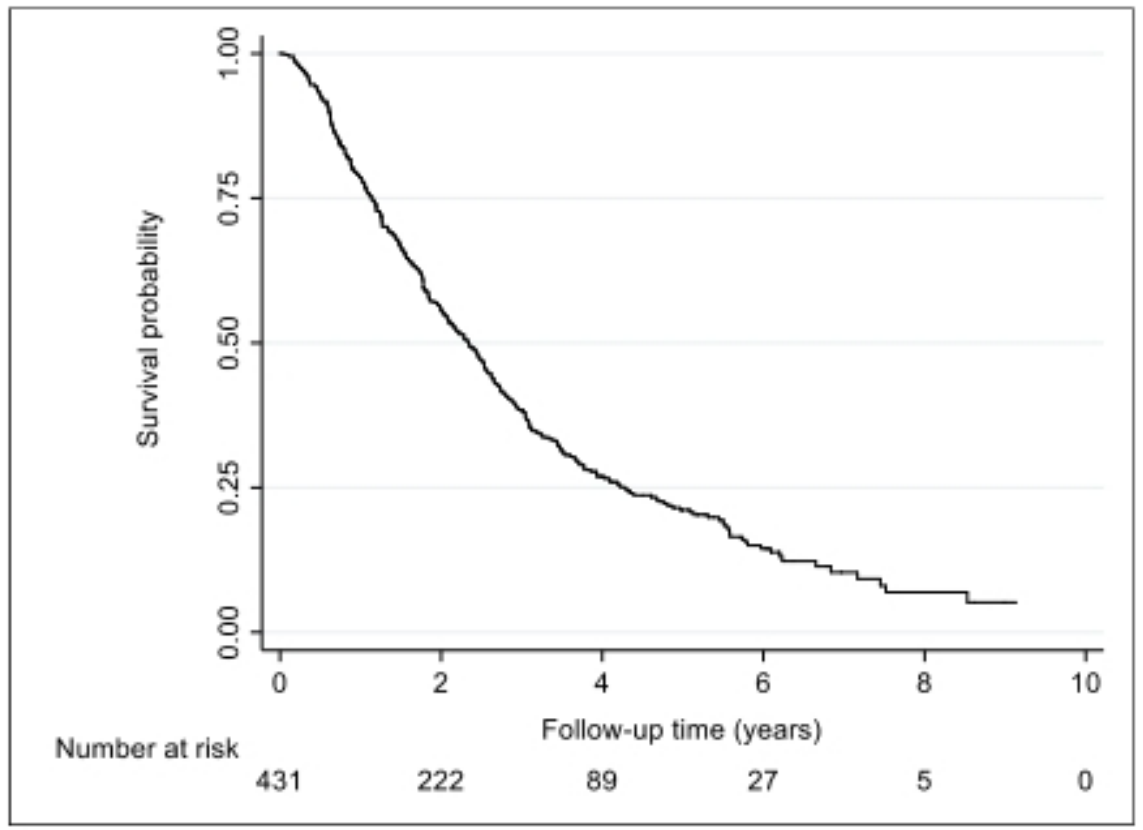

Figure 2. Kaplan Meier curves of overall survival in the overall cohort

OS of the subgroup with the early or intermediate stage (BCLC stage A and B) disease treated with TACE only was 27 months (IQR: 13-44), with the range being 1 day to 110 months.

\section{Safety}

Two patients (0.5\%) had severe complications resulting in death within 4 weeks of initial TACE therapy due to mesenteric ischemia, sepsis, and hepatic decompensation. Other complications following TACE included liver decompensation in sixteen patients (4\%) and renal dysfunction in (2\%), while post-TACE syndrome was the most common at $12 \%$ of the overall cohort [Table 1].

\section{Predictors of survival in the overall cohort}

\section{Univariate analysis}

On univariate analysis, demographic variables associated with survival included ethnicity and country of birth, with Asian patients having a better OS than Caucasian patients $(P=0.001)$ [Table 2A]. In addition, several factors reflective of tumor burden were associated with poor survival; these included the presence of more than three tumors $(P=0.008)$, size of largest HCC (measured in $\mathrm{cm})(P=0.025)$, and BCLC stage $(P<$ $0.0001)$ [Figure $3 \mathrm{~A}$ ]. The presence of macrovascular invasion $(P=0.13)$ and extrahepatic spread $(P=0.42)$ were not related to survival in the small number of patients treated with advanced disease [Table 1 ]. 
Table 2. Univariate analysis of baseline and post treatment variables associated with OS following initial TACE overall cohort (A) and TACE only subgroup (B)

\begin{tabular}{|c|c|c|c|c|}
\hline \multicolumn{5}{|l|}{ (A) Overall cohort $n=431$} \\
\hline Variable & Hazard ratio & Lower $95 \% \mathrm{Cl}$ & Upper 95\%Cl & $P$ value \\
\hline \multicolumn{5}{|l|}{ Demographic } \\
\hline Asian & 0.59 & 0.43 & 0.82 & 0.001 \\
\hline Caucasian & 1.55 & 1.16 & 2.07 & 0.003 \\
\hline HBV & 0.65 & 0.49 & 0.88 & 0.004 \\
\hline Viral hepatitis treatment & 0.47 & 0.22 & 1.01 & 0.048 \\
\hline \multicolumn{5}{|l|}{ Tumour characteristics } \\
\hline Size of largest HCC & 1.05 & 1.00 & 1.09 & 0.025 \\
\hline Single HCC & 0.75 & 0.60 & 0.94 & 0.011 \\
\hline$>3 \mathrm{HCC}$ & 1.39 & 1.09 & 1.77 & 0.008 \\
\hline \multicolumn{5}{|l|}{ Serum Biochemistry } \\
\hline Albumin, $\mathrm{g} / \mathrm{L}$ & 0.94 & 0.92 & 0.96 & $<0.001$ \\
\hline Bilirubin, $\mu \mathrm{mol} / \mathrm{L}$ & 1.02 & 1.01 & 1.03 & $<0.001$ \\
\hline $\mathrm{Na}, \mathrm{mmol} / \mathrm{L}$ & 0.96 & 0.92 & 1.00 & 0.026 \\
\hline $\mathrm{AFP}, \mathrm{ng} / \mathrm{ml}$ & 1.00 & 1.00 & 1.00 & 0.873 \\
\hline \multicolumn{5}{|l|}{ Severity of Liver disease } \\
\hline Encephalopathy & 1.66 & 1.06 & 2.62 & 0.024 \\
\hline Ascites & 2.29 & 1.71 & 3.05 & $<0.001$ \\
\hline Child Pugh score & 1.30 & 1.19 & 1.42 & $<0.001$ \\
\hline Child Pugh grade A & 0.48 & 0.38 & 0.60 & $<0.001$ \\
\hline Child Pugh grade B & 2.06 & 1.63 & 2.60 & $<0.001$ \\
\hline MELD score & 1.04 & 1.01 & 1.07 & 0.009 \\
\hline BCLC stage & 1.35 & 1.14 & 1.60 & $<0.001$ \\
\hline BCLC stage $A$ & 0.66 & 0.53 & 0.83 & $<0.001$ \\
\hline BCLC stage $B$ & 1.44 & 1.15 & 1.81 & 0.001 \\
\hline BCLC stage $C$ & 1.33 & 0.86 & 2.05 & 0.191 \\
\hline \multicolumn{5}{|l|}{ TACE type } \\
\hline CTACE & 0.78 & 0.60 & 1.01 & 0.056 \\
\hline DEB TACE & 1.30 & 0.99 & 1.70 & 0.051 \\
\hline TAE & 1.47 & 0.46 & 4.71 & 0.506 \\
\hline \multicolumn{5}{|l|}{ TACE technique } \\
\hline Non selective & 1.25 & 0.91 & 1.70 & 0.158 \\
\hline Selective & 0.94 & 0.75 & 1.19 & 0.616 \\
\hline Superselective & 0.95 & 0.71 & 1.26 & 0.698 \\
\hline \multicolumn{5}{|l|}{ mRECIST response } \\
\hline $\mathrm{CR}$ & 0.71 & 0.56 & 0.90 & 0.005 \\
\hline PD & 2.04 & 1.43 & 2.92 & $<0.001$ \\
\hline \multicolumn{5}{|l|}{ Combination therapy } \\
\hline Post TACE & 0.53 & 0.38 & 0.74 & $<0.001$ \\
\hline Resection & 0.20 & 0.08 & 0.50 & $<0.001$ \\
\hline Ablation & 0.51 & 0.32 & 0.81 & 0.003 \\
\hline \multicolumn{5}{|c|}{ (B) TACE only subgroup $n=263$} \\
\hline Variable & Hazard ratio & Lower $95 \% \mathrm{Cl}$ & Upper 95\% Cl & $P$ value \\
\hline \multicolumn{5}{|l|}{ Demographic } \\
\hline Asian & 0.58 & 0.39 & 0.87 & 0.008 \\
\hline Caucasian & 1.68 & 1.15 & 2.45 & 0.006 \\
\hline HBV & 0.80 & 0.55 & 1.15 & 0.216 \\
\hline
\end{tabular}




\begin{tabular}{|c|c|c|c|c|}
\hline Viral hepatitis treatment & 0.27 & 0.10 & 0.74 & 0.010 \\
\hline \multicolumn{5}{|l|}{ Tumour characteristics } \\
\hline Size of largest HCC & 1.03 & 0.98 & 1.09 & 0.189 \\
\hline Single HCC & 0.76 & 0.57 & 1.00 & 0.047 \\
\hline$>3 \mathrm{HCC}$ & 1.28 & 0.94 & 1.74 & 0.107 \\
\hline \multicolumn{5}{|l|}{ Serum biochemistry } \\
\hline Albumin, $\mathrm{g} / \mathrm{L}$ & 0.94 & 0.92 & 0.97 & $<0.001$ \\
\hline Bilirubin, $\mu \mathrm{mol} / \mathrm{L}$ & 1.02 & 1.01 & 1.03 & 0.001 \\
\hline $\mathrm{Na}, \mathrm{mmol} / \mathrm{L}$ & 0.98 & 0.93 & 1.03 & 0.354 \\
\hline $\mathrm{AFP}, \mathrm{ng} / \mathrm{ml}$ & 1.00 & 1.00 & 1.00 & 0.675 \\
\hline \multicolumn{5}{|l|}{ Severity of liver disease } \\
\hline Encephalopathy & 1.52 & 0.90 & 2.56 & 0.110 \\
\hline Ascites & 1.91 & 1.35 & 2.72 & $<0.001$ \\
\hline Child Pugh score & 1.32 & 1.19 & 1.47 & $<0.001$ \\
\hline Child Pugh grade $\mathrm{A}$ & 0.46 & 0.34 & 0.61 & $<0.001$ \\
\hline Child Pugh grade B & 2.14 & 1.60 & 2.86 & $<0.001$ \\
\hline MELD score & 1.04 & 1.01 & 1.08 & 0.020 \\
\hline BCLC stage & 1.50 & 1.14 & 1.99 & 0.004 \\
\hline$B C L C$ stage $A$ & 0.66 & 0.50 & 0.88 & 0.004 \\
\hline BCLC stage $B$ & 1.50 & 1.14 & 1.99 & 0.004 \\
\hline BCLC stage $C$ & $\mathrm{~N} / \mathrm{A}$ & N/A & $\mathrm{N} / \mathrm{A}$ & $\mathrm{N} / \mathrm{A}$ \\
\hline \multicolumn{5}{|l|}{ TACE type } \\
\hline CTACE & 0.85 & 0.61 & 1.18 & 0.320 \\
\hline DEB TACE & 1.20 & 0.86 & 1.67 & 0.282 \\
\hline TAE & 0.72 & 0.10 & 5.37 & 0.745 \\
\hline \multicolumn{5}{|l|}{ TACE technique } \\
\hline Non selective & 1.14 & 0.77 & 1.70 & 0.501 \\
\hline Selective & 0.92 & 0.68 & 1.23 & 0.563 \\
\hline Superselective & 1.14 & 0.80 & 1.64 & 0.457 \\
\hline \multicolumn{5}{|l|}{ mRECIST response } \\
\hline CR & 0.83 & 0.61 & 1.13 & 0.228 \\
\hline PD & 1.83 & 1.17 & 2.86 & 0.007 \\
\hline \multicolumn{5}{|l|}{ Combination therapy } \\
\hline Post TACE & N/A & N/A & N/A & N/A \\
\hline Resection & N/A & N/A & $\mathrm{N} / \mathrm{A}$ & $\mathrm{N} / \mathrm{A}$ \\
\hline Ablation & N/A & N/A & N/A & N/A \\
\hline
\end{tabular}

AFP: Alpha fetoprotein; BCLC: Barcelona clinic liver cancer; CTACE: conventional TACE; CI: confidence interval; DEBTACE: drug eluting bead TACE; HBV: hepatitis B virus; mRECIST: modified response evaluation criteria in solid tumors; N/A: not applicable; OS: overall survival; PD: progressive disease; TACE: transarterial chemoembolization; TAE: transarterial embolization.

Notably, TACE technique type, selectivity of hepatic artery cannulation, and type of embolisation material deployed did not affect survival [Table 2A]. Combination treatment with TACE either with surgical resection $(P<0.001)$ or ablation $(P=0.003)$ resulted in improved OS [Table $2 \mathrm{~A}$ ]. Multiple biochemical parameters before initial TACE were associated with OS, including serum albumin $(P<0.0001)$, bilirubin $(P$ $<0.0001)$, and sodium $(P=0.026)$. Scores that incorporate these variables were also significantly associated with OS including the Child-Pugh grade [Figure 3B] $(P \leq 0.001)$ and MELD score $(P=0.009)$ [Table 2A].

\section{Multivariate analysis}

On multivariate analysis, ascites pre-TACE and larger HCC were independent predictors of poor survival, whereas HBV infection as the etiology of HCC, higher serum albumin pre-treatment, and single HCC were 


\section{A}

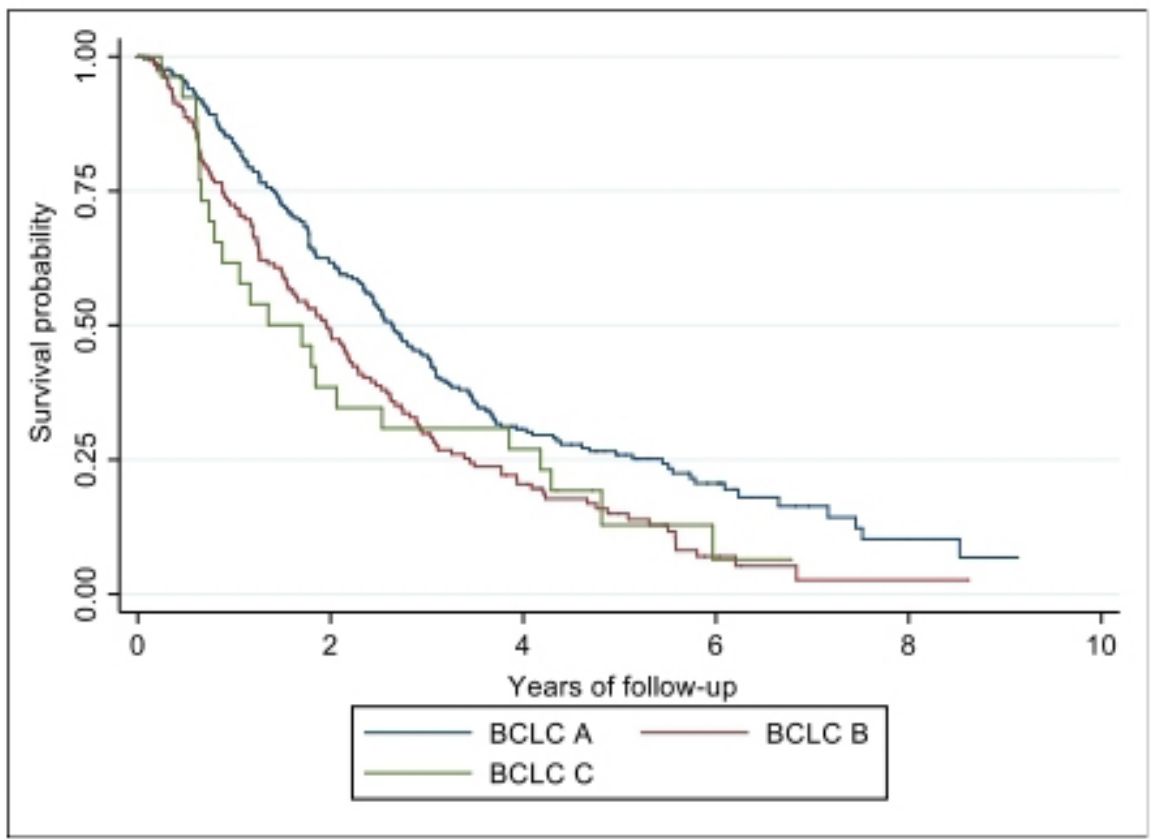

\section{B}

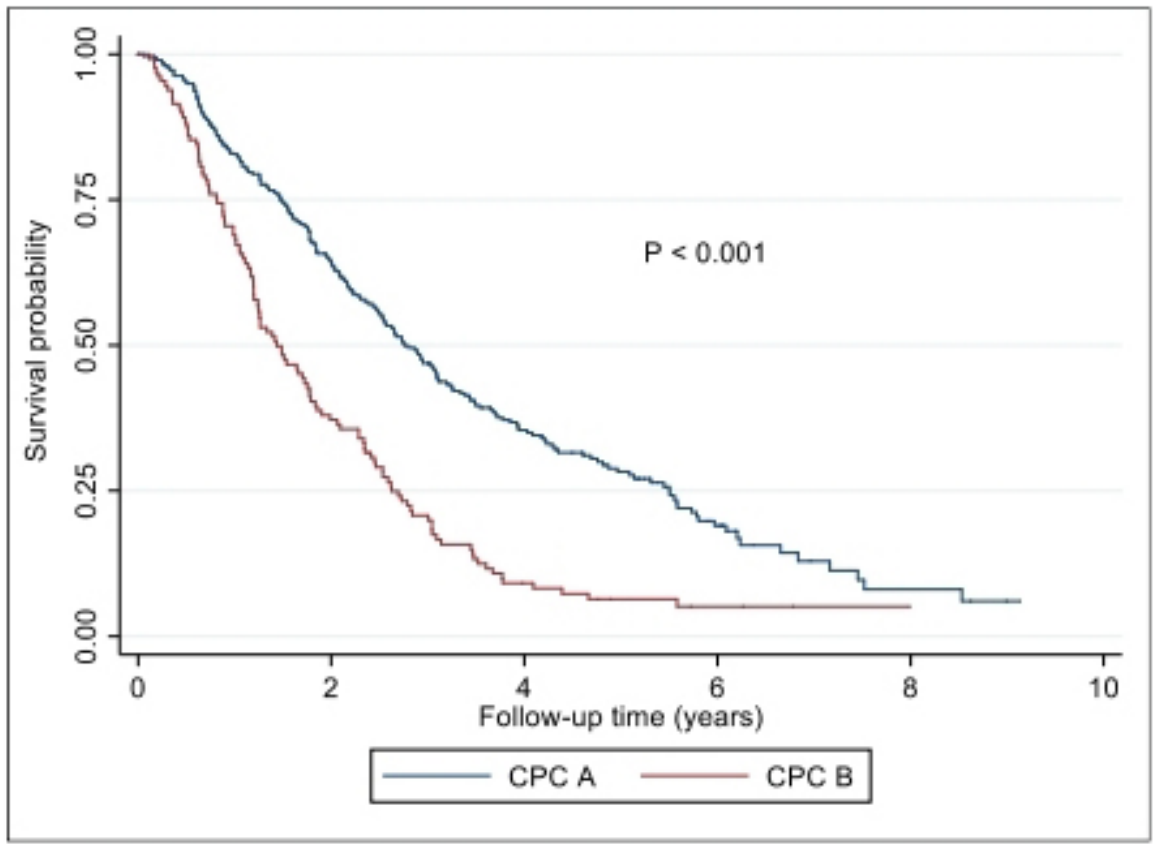

Figure 3. Kaplan Meier curves of $B C L C$ stage (A) and Child Pugh (B) correlation with overall survival in the overall cohort. $B C L C$ : Barcelona clinic liver cancer; CPC A: child Pugh class A; CPC B: child Pugh class B.

associated with improved survival after TACE [Table 3A]. 
Table 3. Multivariate analysis - pre-TACE variables associated with overall survival in the (A) overall cohort and (B) the TACE only subgroup

\begin{tabular}{|c|c|c|c|c|c|c|c|}
\hline \multicolumn{8}{|c|}{ (A) Overall cohort $n=431$} \\
\hline Variable & \multicolumn{2}{|c|}{ Hazard ratio } & \multicolumn{2}{|c|}{ Lower 95\% Cl } & \multicolumn{2}{|c|}{ Upper $95 \% \mathrm{Cl}$} & $P$ value \\
\hline Albumin & \multicolumn{2}{|l|}{0.95} & \multicolumn{2}{|l|}{0.92} & \multicolumn{2}{|c|}{0.97} & $<0.0001$ \\
\hline Ascites & \multicolumn{2}{|c|}{2.33} & \multicolumn{2}{|l|}{1.61} & \multicolumn{2}{|c|}{3.38} & $<0.0001$ \\
\hline HBV & \multicolumn{2}{|l|}{0.61} & \multicolumn{2}{|l|}{0.43} & \multicolumn{2}{|c|}{0.86} & 0.005 \\
\hline Single HCC & \multicolumn{2}{|c|}{0.70} & \multicolumn{2}{|l|}{0.54} & \multicolumn{2}{|c|}{0.91} & 0.007 \\
\hline Size of largest $\mathrm{HCC}$ & \multicolumn{2}{|l|}{1.06} & 1.00 & & \multicolumn{2}{|l|}{1.11} & 0.036 \\
\hline \multicolumn{8}{|c|}{ (B) TACE only subgroup $n=263$} \\
\hline Variable & Hazard ratio & \multicolumn{2}{|l|}{ Lower $95 \% \mathrm{Cl}$} & \multicolumn{2}{|c|}{ Upper $95 \% \mathrm{Cl}$} & \multicolumn{2}{|c|}{$P$ value } \\
\hline Albumin & 0.94 & \multicolumn{2}{|l|}{0.91} & \multicolumn{2}{|c|}{0.97} & \multicolumn{2}{|c|}{$<0.0001$} \\
\hline Ascites & 1.70 & \multicolumn{2}{|l|}{1.15} & \multicolumn{2}{|c|}{2.53} & \multicolumn{2}{|c|}{0.008} \\
\hline Caucasian ethnicity & 1.59 & \multicolumn{2}{|l|}{1.03} & \multicolumn{2}{|c|}{2.48} & \multicolumn{2}{|c|}{0.038} \\
\hline$>3 \mathrm{HCC}$ & 1.53 & \multicolumn{2}{|l|}{1.06} & \multicolumn{2}{|c|}{2.21} & \multicolumn{2}{|c|}{0.024} \\
\hline
\end{tabular}

Cl: Confidence interval; HBV: hepatitis B virus; HCC: hepatocellular carcinoma; OS: overall survival; TACE: transarterial chemoembolization.

\section{Predictors of survival in the TACE only treatment subgroup \\ Univariate analysis}

Several variables were associated with OS on univariate analysis of the refined cohort of 263 patients, many of which were similar to those identified in the overall group. These include ethnicity, and pre-treatment serum albumin and bilirubin $(P \leq 0.001)$ [Table $2 \mathrm{~B}$ ]. As expected, both Child-Pugh B status and BCLC stage $\mathrm{B}$ disease negatively impacted survival compared to Child-Pugh A and BCLC stage A patients respectively. Of the parameters reflective of tumor burden, only a single HCC was significant $(P=0.047)$ [Table $2 \mathrm{~B}]$. TACE technique and selectivity of TACE did not influence OS [Table 2B].

\section{Multivariate analysis}

On multivariate analysis of the TACE only subgroup, higher serum albumin $(P<0.001)$ was associated with improved OS, while pre-treatment ascites $(P=0.008)$, greater than 3 tumour nodules before TACE $(P=$ $0.024)$ and Caucasian ethnicity $(P=0.038)$ were significantly associated with worse OS [Table 3B].

\section{DISCUSSION}

TACE is the recommended treatment for intermediate-stage HCC. However, significant variation in survival outcomes is observed following treatment despite increased adoption of multidisciplinary-based review to improve patient selection. This study is the first large multicenter Australian cohort study to evaluate pre-treatment prognostic factors associated with survival in HCC patients undergoing TACE. Our study identified that markers of hepatic function and tumor burden independently predicted the overall survival of the cohort.

Notably, markers of hepatic function before TACE were strongly predictive of survival with higher serum albumin associated with lower risk of post-treatment mortality in both the overall cohort and refined subgroup, while pre-treatment ascites carried the greatest risk of a poor outcome. These findings are consistent with previous cohort studies that have found decompensation, particularly ascites, had one of the highest risks of post TACE decompensation and poorer survival ${ }^{[19]}$. 
Multivariate analysis also highlighted the role of tumor burden in predicting poorer outcomes following TACE. Tumor size and number were significant in the overall cohort, as patients with single HCC classified as BCLC stage A disease $(P=0.007)$ had better survival, compared to patients with multifocal and larger HCC stage (i.e., BCLC stage $\mathrm{B})$ disease $(P=0.036)$. Tumor number and size, along with MVI or EHS, have been previously described as surrogate markers of tumor biology and aggressiveness and have been linked to increased risk of treatment-related complications and poorer survival outcomes ${ }^{[20-22]}$.

The inherent heterogeneity of patients with unresectable HCC in relation to the variations in tumor burden and underlying hepatic dysfunction significantly influences patient outcomes after TACE. It partly explains the wide range of OS we observed in our cohort, although the median survival of the group of 28 months was considerably higher than the 19-20 months reported in an early meta-analysis of randomised controlled studies $^{[10,12]}$ and recently updated systematic review ${ }^{[1,23]}$. The difference in survival outcomes may be due to several factors, including the routine involvement of MDT in decision making across all sites, the utilisation of aggressive treatment modalities such as surgical resection and ablation in combination with TACE, and the inclusion of a significant proportion of BCLC stage A along with a small number of BCLC C stage patients [Table 1]. Nevertheless, we also observed a respectable 27 months median survival in the subgroup of patients with similar characteristics of cohort studies that form the basis of current TACE guidelines, those with BCLC stage A or B disease treated with TACE alone $\mathrm{e}^{[1,1,16,19]}$.

A novel finding of this study was that the etiology of liver disease and ethnicity were prognostic markers of survival. Specifically, hepatitis B-related liver disease was an independent predictor of lower mortality $(P=$ 0.004 ) with a median OS of 34 months (IQR: 15-72) compared to 26 months in the non-HBV group (IQR: 13-45). This finding is consistent with previous studies identifying a prognostic role for HBV in patients with HCC. It may relate to associated factors such as participation in active HCC surveillance programs that have a positive effect on survival primarily in those with earlier stage disease ${ }^{[1724,25]}$. This is reflected in the more favourable clinical characteristics of patients with HBV liver disease in our overall cohort compared to non-HBV subjects including, younger age at diagnosis $(P<0.001)$, better liver reserve with lower rates of cirrhosis ( $83 \%$ vs. 94\%), higher serum albumin $(P=0.004)$, lower INR $(P=0.004)$, and bilirubin $(P=0.015)$.

In addition, patients with HBV liver disease had lower rates of concomitant HCV infection $(P=0.001)$ and ETOH abuse $(P=0.001)$. However, further subgroup analysis by other co-factors, including alcohol, is limited due to the relatively small number of patients within each variable (data not shown). Commensurate with this, Caucasian ethnicity was associated with poorer TACE related outcomes on multivariate analysis in the subgroup of patients receiving TACE only treatment, likely due to lower rates of HBV related liver disease $(P<0.0001)$ and higher rates of multifocal HCC $(27 \%$ vs. $19 \%)$ and poorer hepatic reserve (with Child Pugh B in $33 \%$ vs. $13 \%$ of non-caucasian patients).

Variables beyond BCLC stage can also determine treatment outcomes, such as variations in TACE administration and combination therapies based on the stage migration principle; due to tumour location or comorbidities that is not amenable for recommended first-line treatment ${ }^{[26]}$. Our study found that the selectivity and mode of TACE utilized did not impact overall survival regardless of which cohort was analysed. Moreover, there was no impact on survival on multivariate analysis in patients that underwent post TACE surgical resection or ablation, although better outcomes were noted from combination therapies on univariate analysis in the overall cohort [Table $2 \mathrm{~B}]$.

Key strengths of our study include the cohort size and multicenter design and analysis of real-world data of BCLC stage guided treatment, reflecting current established standards of care globally. In particular, the 
utilisation HCC surveillance programs, MDT tumor board meetings, TACE techniques, and mRECIST assessment of response across the six participating tertiary centres, increased the homogeneity of criteria used for diagnosis, staging, and treatment allocation. Furthermore, a significant number of patients were treated within the guidelines for TACE therapy according to the BCLC staging system, with the addition of BCLC stage A and a smaller number of patients with BCLC stage C HCC, consistent with current realworld international practice ${ }^{[16,27-30]}$.

One important reason as to why BCLC A subjects received TACE was via the stage migration principle in which subjects with early-stage disease were deemed unsuitable for curative treatment options of surgery or local ablation. In particular, those with unresectable HCC adjacent to major vessels or in close proximity to gallbladder or bowel received TACE rather than ablation to avoid the heat sink effect and adjacent soft tissue damage respectively ${ }^{[1,16]}$. In addition, in a minority of cases, local treatment preferences for TACE over ablation in BCLC A subjects were a factor. Furthermore, we had a subgroup of patients that underwent sequential combination therapy with TACE following downstaging of the initial HCC; this included surgical resection and ablation. Patients that underwent TACE as a bridge to liver transplantation were excluded from the analysis. Most importantly, the decision to proceed with TACE was made after a multi-disciplinary team review in all cases.

Of those that underwent post-TACE combination therapy, there was a significant improvement in overall survival on univariate analysis $(P<0.0001)$ in patients that underwent post-TACE surgical resection and ablation $(P=0.003)$. However, the subgroup compromised of small patient numbers precluding more detailed multivariate analysis and applicability with only $4 \%(n=16)$ and $8 \%(n=35)$ of patients undergoing resection or ablation respectively.

Similarly, a small minority (6\%) BCLC-C cases also underwent TACE therapy $(n=27)$. The rationale for using TACE in the majority of these patients was for symptom control from significant tumor burden. Various scenarios likely contributed to TACE being offered in these cases, including limited metastases with non-occlusive vascular involvement in 16 patients. There was no significant difference in overall survival on univariate analysis of the BCLC-C patients $(P=0.191)$, likely reflective of the small number of patients in this subgroup. However, surrogate markers of advanced HCC, including size and number of HCC, were significant on multivariate analysis in the overall and TACE only subgroup.

Still, the study was limited by the retrospective collection of data and the reliance on accurate records and subjectively assessed parameters such as ascites and performance status incorporated into the Child-Pugh grade and BCLC stage respectively. As a result, this study is subject to selection bias relative to the selection of patients analysed in this cohort. Finally, survival status was known in only around $80 \%$ of the cohort, with the remainder of subjects censored at the last clinical follow-up. However, there were no clinically significant differences between those in whom survival status was known and not known (data not shown).

\section{Conclusion}

In this large multi-centered real-world study of HCC patients undergoing TACE, we found that patients with more advanced liver disease associated with ascites and/or greater tumour burden have poorer outcomes following treatment. Such findings provide a better understanding of the variation in survival after TACE and could be potentially helpful in selecting and counselling patients undergoing this therapy. 


\section{DECLARATIONS}

\section{Acknowledgements}

We thank Mr. Paul O’Neil, Ms. Virginia Bird, Ms. Pam Crest, Ms. Angela Li, Ms. Anne Dowling, and Ms. Carol McCrae for their assistance in data collation.

\section{Authors' contributions}

Contributed to the study design, conception, data analysis, and interpretation: Mishra G, Dev A, Roberts SK Performed data acquisition: Mishra G

Performed statistical analysis: Paul E

Provided administrative, technical and material support: Roberts SK, Lubel J, Gow P, Sood S, Thompson A All authors contributed to the drafting and/or review of the manuscript.

\section{Availability of data and materials}

All supplementary data is available for review upon request submitted to the corresponding author.

\section{Financial support and sponsorship}

Mishra G was supported by an Australian Government Research Training Program (RTP) Scholarship, Monash University, Melbourne, Australia.

\section{Conflicts of interest}

All authors declared that there are no conflicts of interest.

\section{Ethical approval and consent to participate}

Not applicable.

\section{Consent for publication}

Not applicable.

\section{Copyright}

(c) The Author(s) 2021.

\section{REFERENCES}

1. Bray F, Ferlay J, Soerjomataram I, Siegel RL, Torre LA, Jemal A. Global cancer statistics 2018: GLOBOCAN estimates of incidence and mortality worldwide for 36 cancers in 185 countries. CA Cancer J Clin 2018;68:394-424. DOI PubMed

2. Torre LA, Siegel RL, Ward EM, Jemal A. Global cancer incidence and mortality rates and trends - an update. Cancer Epidemiol Biomarkers Prev 2016;25:16-27. DOI PubMed

3. El-Serag HB, Marrero JA, Rudolph L, Reddy KR. Diagnosis and treatment of hepatocellular carcinoma. Gastroenterology 2008;134:1752-63. DOI PubMed

4. Sherman M. Staging for hepatocellular carcinoma: complex and confusing. Gastroenterology 2014;146:1599-602. DOI PubMed

5. Marrero JA, Kudo M, Bronowicki JP. The challenge of prognosis and staging for hepatocellular carcinoma. Oncol 2010;15 Suppl 4:23-33. DOI PubMed

6. Llovet JM, Brú C, Bruix J. Prognosis of hepatocellular carcinoma: the BCLC staging classification. Semin Liver Dis 1999;19:329-38. DOI PubMed

7. Forner A, Reig ME, de Lope CR, Bruix J. Current strategy for staging and treatment: the BCLC update and future prospects. Semin Liver Dis 2010;30:61-74. DOI PubMed

8. Llovet JM, Zucman-Rossi J, Pikarsky E, et al. Hepatocellular carcinoma. Nat Rev Dis Primers 2016;2:16018. DOI PubMed

9. Raoul JL, Sangro B, Forner A, et al. Evolving strategies for the management of intermediate-stage hepatocellular carcinoma: available evidence and expert opinion on the use of transarterial chemoembolization. Cancer Treat Rev 2011;37:212-20. DOI PubMed

10. Llovet JM, Bruix J. Systematic review of randomized trials for unresectable hepatocellular carcinoma: chemoembolization improves survival. Hepatology 2003;37:429-42. DOI PubMed

11. Roberts SK, Gazzola A, Lubel J, et al; Melbourne Liver Group. Treatment choice for early-stage hepatocellular carcinoma in realworld practice: impact of treatment stage migration to transarterial chemoembolization and treatment response on survival. Scand $J$ Gastroenterol 2018;53:1368-75. DOI PubMed

12. Lo CM, Ngan H, Tso WK, et al. Randomized controlled trial of transarterial lipiodol chemoembolization for unresectable 
hepatocellular carcinoma. Hepatology 2002;35:1164-71. DOI PubMed

13. Llovet JM, Real MI, Montaña X, et al. Arterial embolisation or chemoembolisation versus symptomatic treatment in patients with unresectable hepatocellular carcinoma: a randomised controlled trial. Lancet 2002;359:1734-9. DOI PubMed

14. Takayasu K, Arii S, Kudo M, et al. Superselective transarterial chemoembolization for hepatocellular carcinoma. Validation of treatment algorithm proposed by Japanese guidelines. J Hepatol 2012;56:886-92. DOI PubMed

15. Burrel M, Reig M, Forner A, et al. Survival of patients with hepatocellular carcinoma treated by transarterial chemoembolisation (TACE) using Drug Eluting Beads. Implications for clinical practice and trial design. J Hepatol 2012;56:1330-5. DOI PubMed

16. Marrero JA, Kulik LM, Sirlin CB, et al. Diagnosis, staging, and management of hepatocellular carcinoma: 2018 practice guidance by the American association for the study of liver diseases. Hepatology 2018;68:723-50. DOI PubMed

17. Mgaieth S, Kemp W, Gow P, et al. Impact of viral hepatitis aetiology on survival outcomes in hepatocellular carcinoma: a large multicentre cohort study. J Viral Hepat 2017;24:982-9. DOI PubMed

18. Clark TW. Complications of hepatic chemoembolization. Semin Intervent Radiol 2006;23:119-25. DOI PubMed PMC

19. Raoul JL, Forner A, Bolondi L, Cheung TT, Kloeckner R, de Baere T. Updated use of TACE for hepatocellular carcinoma treatment: how and when to use it based on clinical evidence. Cancer Treat Rev 2019;72:28-36. DOI PubMed

20. Finn RS, Zhu AX, Farah W, et al. Therapies for advanced stage hepatocellular carcinoma with macrovascular invasion or metastatic disease: a systematic review and meta-analysis. Hepatology 2018;67:422-35. DOI PubMed

21. Katayama K, Imai T, Abe Y, et al. Number of nodules but not size of hepatocellular carcinoma can predict refractoriness to transarterial chemoembolization and poor prognosis. J Clin Med Res 2018;10:765-71. DOI PubMed PMC

22. Lee YH, Hsu CY, Huang YH, et al. Vascular invasion in hepatocellular carcinoma: prevalence, determinants and prognostic impact. $J$ Clin Gastroenterol 2014;48:734-41. DOI PubMed

23. Lencioni R, de Baere T, Soulen MC, Rilling WS, Geschwind JF. Lipiodol transarterial chemoembolization for hepatocellular carcinoma: a systematic review of efficacy and safety data. Hepatology 2016;64:106-16. DOI PubMed

24. Kwak HW, Park JW, Nam BH, et al. Clinical outcomes of a cohort series of patients with hepatocellular carcinoma in a hepatitis B virus-endemic area. J Gastroenterol Hepatol 2014;29:820-9. DOI PubMed

25. Hong TP, Gow PJ, Fink M, et al. Surveillance improves survival of patients with hepatocellular carcinoma: a prospective populationbased study. Med J Aust 2018;209:348-54. DOI PubMed

26. Díaz-González Á, Reig M, Bruix J. Treatment of hepatocellular carcinoma. Dig Dis 2016;34:597-602. DOI PubMed

27. Association for the Study of the Liver, European Association for the Study of the Liver. EASL clinical practice guidelines: management of hepatocellular carcinoma. J Hepatol 2018;69:182-236. DOI

28. Piscaglia F, Ogasawara S. Patient selection for transarterial chemoembolization in hepatocellular carcinoma: importance of benefit/risk assessment. Liver Cancer 2018;7:104-19. DOI PubMed PMC

29. Sangiovanni A, Colombo M. Treatment of hepatocellular carcinoma: beyond international guidelines. Liver Int 2016;36 Suppl 1:124-9. DOI PubMed

30. Guarino M, Tortora R, de Stefano G, et al; Progetto Epatocarcinoma Campania Group. Adherence to barcelona clinic liver cancer guidelines in field practice: results of progetto epatocarcinoma campania. J Gastroenterol Hepatol 2018;33:1123-30. DOI PubMed 